\title{
I. Özdemir
}

\section{A numerical solution framework for simultaneous peeling of thin elastic strips from a rigid substrate}

Received: 2 July 2016 / Revised: 26 October 2016 / Published online: 17 January 2017

(C) Springer-Verlag Wien 2017

\begin{abstract}
Simultaneous peeling of multiple strips is commonly observed particularly at small-scale detachment processes. Although theoretical treatment of this problem is addressed, numerical solution procedures for geometrically arbitrary multiple-peeling problems are still missing. In this paper, a finite element-based numerical solution procedure for 3-D large displacement multiple-peeling problems is presented. Loading/unloading of peeling strips are expressed in the form of optimality conditions, and the current positions of the peeling fronts are determined locally adapting the multiplicative decomposition and the return mapping algorithm of finite strain plasticity theories. Within an incremental-iterative solution framework, peeling fronts and the current position of other nodes are determined in a staggered way instead of using an active set-based solution algorithm. The effectiveness of the approach is demonstrated by a series of example problems including multiple peeling of an assembly of randomly oriented strips.
\end{abstract}

\section{Introduction}

Biological adhesives such as a Gecko's foot and spider web anchorages have been the focus of many recent studies primarily due to their outstanding and unique adhesion/detachment properties [1,2]. By splitting the contact among discrete localized regions through a hierarchical structure, a Gecko can achieve adhesion strengths much higher than the animal's body weight. Similarly, spider web anchorages have a hierarchical fibre structure typically branching from a superstructure. The geometry of these branched structures takes different morphologies for different purposes, such as staple-pin-like attachments for anchorage and dendritic structures for prey-capture [3].

Kendall's analysis on thin-film peeling [4] has been very instrumental to understand the peeling mechanics of biological adhesives. With certain extensions of Kendall's analysis [5,6], different physical aspects of the adhesion and detachment of a Gecko's foot could be explained and predicted. However, as far as web anchorage-type geometries are concerned, the mechanics of peeling involves multiple peeling of different fibres simultaneously. To address this, Pugno [7] developed a theory for multiple peeling which is in fact based on Kendall's work. Closed-form solutions for relatively simpler layouts such as V-type double-peeling problems and axisymmetric membrane problems were derived [8]. Furthermore, these closed-form solutions are also compared with detailed finite element analysis in the context of hierarchical multiple-peeling configurations [9]. Recently, a numerical implementation of the multiple-peeling theory has been presented to address geometrically more complex problems [10]. However, the problems considered reveal that the solution procedure makes use of symmetry in certain cases and the important finding of Pugno [7] allowing superposition of single-peeling solution for certain (limited) geometries of multiple-peeling configurations. Therefore, for geometrically complicated 3-D multiple-peeling problems, a reliable numerical solution procedure is essential.

İ. Özdemir $(\varangle)$

Department of Civil Engineering, İzmir Institute of Technology, Gülbahçe Kampüsü, Urla, Izmir, Turkey

E-mail: izzetozdemir@iyte.edu.tr 
Departing from this fact, in this paper, a finite element-based numerical solution framework for 3-D large displacement multiple-peeling problems is presented. Borrowing the concept of multiplicative decomposition from finite strain inelasticity theories, an intermediate stress-free peeled configuration is introduced. Treating energy balance as the peeling criterion, a plasticity-like return mapping algorithm is used and peeling of each strip is effectively updated. This local algorithm is embedded within the global incremental-iterative solution procedure in such a way that the update of peeling fronts is followed by the update of the current position of other nodes. The effectiveness of this staggered solution algorithm is demonstrated by example problems.

The paper is organized as follows. In the next section, the multiple-peeling problem is defined formally including some details of the global solution algorithm. Then, the element-level formulation, the adapted return mapping algorithm and how it is embedded within the global framework are detailed in Sect. 3. In Sect. 4 , the effectiveness of the approach is demonstrated through a set of examples before the paper is closed by a conclusion and outlook section.

\section{Problem definition}

Multiple peeling typically occurs in systems where multiple deformable strips meet at a point or fibres branch off a major component of the system. Referring to Fig. 1, the geometry of each member in the system is described by a starting node (point) and an end node (point) and a peeling direction $\mathbf{n}_{p}$ for the end which is attached to the rigid substrate. Obviously, there might be some members which are not attached to the rigid substrate. In what follows, the end/junction points which are not attached to the substrate are called ordinary end/junction points and the terms node and point are used interchangeably. Similarly, elements without a peeling end are classified as ordinary elements. The structure is assumed to be under the action of externally applied displacements at an arbitrary number of junction points, and externally applied forces are not included in the discussion. Furthermore, it is assumed that the members can sustain only tensile forces. Therefore, the deformed configuration of the system should be such that each strip is stretched or stretched and peeling or at its original length. This imposes restrictions on the problems which can be analysed and particularly excludes the configurations where members get compressed. The undeformed and deformed configurations of such a structure are shown in Fig. 1. The undeformed and deformed position vectors of end points are shown by $\mathbf{X}^{i}$ and $\mathbf{x}^{i}$ with the superscript standing for the node number, respectively. The current position vector of the ends which are attached to the substrate is expressed as

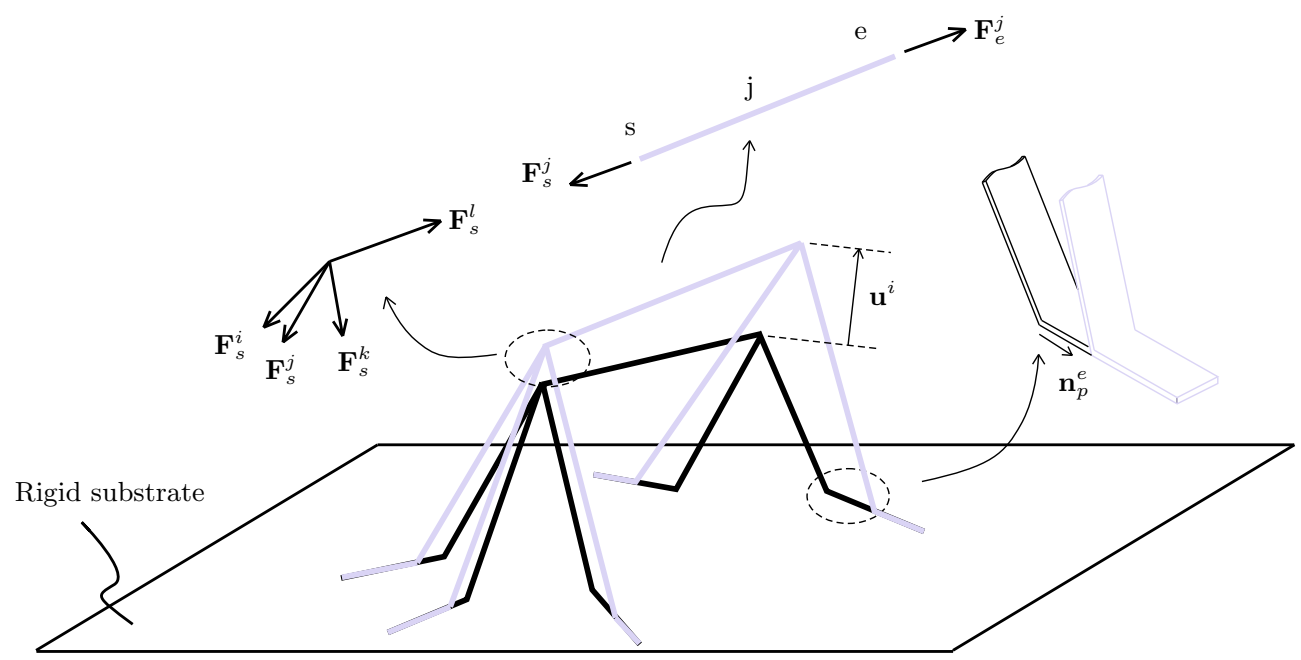

Fig. 1 A structure with multiple peeling in undeformed and deformed configurations. The forces acting on an ordinary junction point and the details of a peeling front are shown as well 


$$
\mathbf{x}^{i}=\mathbf{X}^{i}+l_{p}^{i} \mathbf{n}_{p}^{i}
$$

where $l_{p}^{i}$ is the total peeling of the corresponding element. For ordinary junction points, the two position vectors are connected by the displacement vector $\mathbf{u}^{i}$ through $\mathbf{x}^{i}=\mathbf{X}^{i}+\mathbf{u}^{i}$.

In order to identify the deformed configuration, unknown $l_{p}^{i}$ values and the components of the displacement vectors $\mathbf{u}^{i}$ of ordinary junction points have to be determined. In the deformed state, ignoring bending stiffness of the fibres, in each member a uniform state of axial stress develops. Referring to Fig. 1, for each member the nodal forces can be written as

$$
\mathbf{F}_{e}^{j}=A \sigma \mathbf{n}^{e}, \quad \mathbf{F}_{s}^{j}=-\mathbf{F}_{e}^{j},
$$

where $\sigma$ is the Cauchy stress and $A$ is the current cross-sectional area of the member. The subscripts $s$ and $e$ stand for starting point and end point of an element, respectively. The unit vector $\mathbf{n}^{e}$ is pointing to the end point of the element in the deformed configuration. Since the system is in equilibrium, the nodal forces meeting at an ordinary junction point should sum up to zero. In other words, the out of balance nodal force $\mathbf{R}_{i}$ at every ordinary junction point should satisfy

$$
\mathbf{R}^{i}=\sum_{j=1}^{k} \mathbf{F}_{s, e}^{j}=\mathbf{0},
$$

where the summation is over the elements sharing the junction point of interest. Due to the use of a nonlinear strain measure (see Sect. 3) and the fact that $\mathbf{n}^{e}$ depends on the current configuration, Eq. (3) is nonlinear and solved using the Newton-Raphson method. In this iterative solution procedure, the nonlinear set of equations is linearized around a known state as

$$
\underline{R}\left(\underline{u}_{k}\right)+\left.\frac{\partial \underline{R}}{\partial \underline{u}}\right|_{k} \delta \underline{u}=\underline{R}\left(\underline{u}_{k}\right)+\underline{K}\left(\underline{u}_{k}\right) \delta \underline{u}=\underline{0},
$$

where $\underline{R}$ and $\underline{K}$ are the nodal residuum column and the tangent matrix of the system assembled in the usual finite element sense from element contributions. The solution for the nodal displacements $\underline{u}_{k+1}$ at iteration $k+1$ is obtained through

$$
\begin{aligned}
\underline{K}\left(\underline{u}_{k}\right) \delta \underline{u} & =-\underline{R}\left(\underline{u}_{k}\right), \\
\underline{u}_{k+1} & =\underline{u}_{k}+\delta \underline{u},
\end{aligned}
$$

where $\delta \underline{u}$ is the column of iterative corrections. This procedure is repeated until the norm of the residuum column gets smaller than a user-specified convergence tolerance. A detailed treatment of Newton-Raphson method in the context of nonlinear solid/structural mechanics can be found in [11-13], among others.

As explained in the next section, peeling of a specific element $\left(l_{p}^{i}\right)_{k+1}$ is determined at element level on the basis of an energy balance between the surface energy change and strain energy change in the member.

\section{Element-level formulation}

In Fig. 2, a single element with a peeling end is isolated and shown in undeformed and deformed configurations. The current positions of the starting and end points of the member are written as

$$
\begin{aligned}
\mathbf{x}^{s} & =\mathbf{X}^{s}+\mathbf{u}^{s}, \\
\mathbf{x}^{e} & =\mathbf{X}^{e}+l_{p}^{e} \mathbf{n}_{p}^{e} .
\end{aligned}
$$

The member experiences both peeling and stretching during this motion. Obviously, stress develops in the member due to stretching and one can conveniently decompose the total stretch of the member into a stressproducing elastic part $\lambda^{e}$ and a peeling part $\lambda^{p}$ mapping the fibre to the stress-free intermediate configuration, see Fig. 3. Therefore, the following equalities hold:

$$
\begin{aligned}
& l=\lambda l_{0}, \\
& l=\lambda_{e} \lambda_{p} l_{0}, \\
& l=\lambda_{e} l^{*} \quad \text { with } l^{*}=\lambda_{p} l_{0} \text { and } l^{*}=l_{0}+l_{p},
\end{aligned}
$$




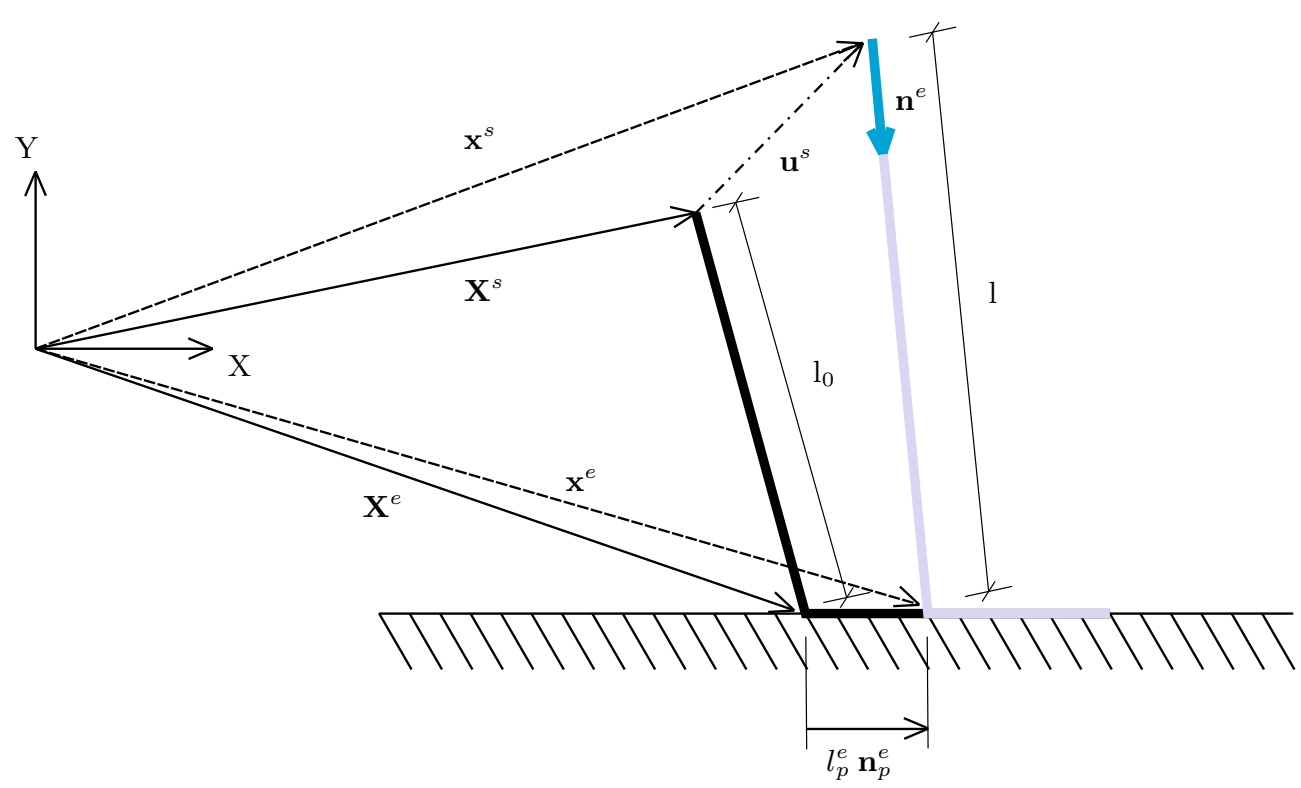

Fig. 2 A single strip in undeformed and deformed configurations

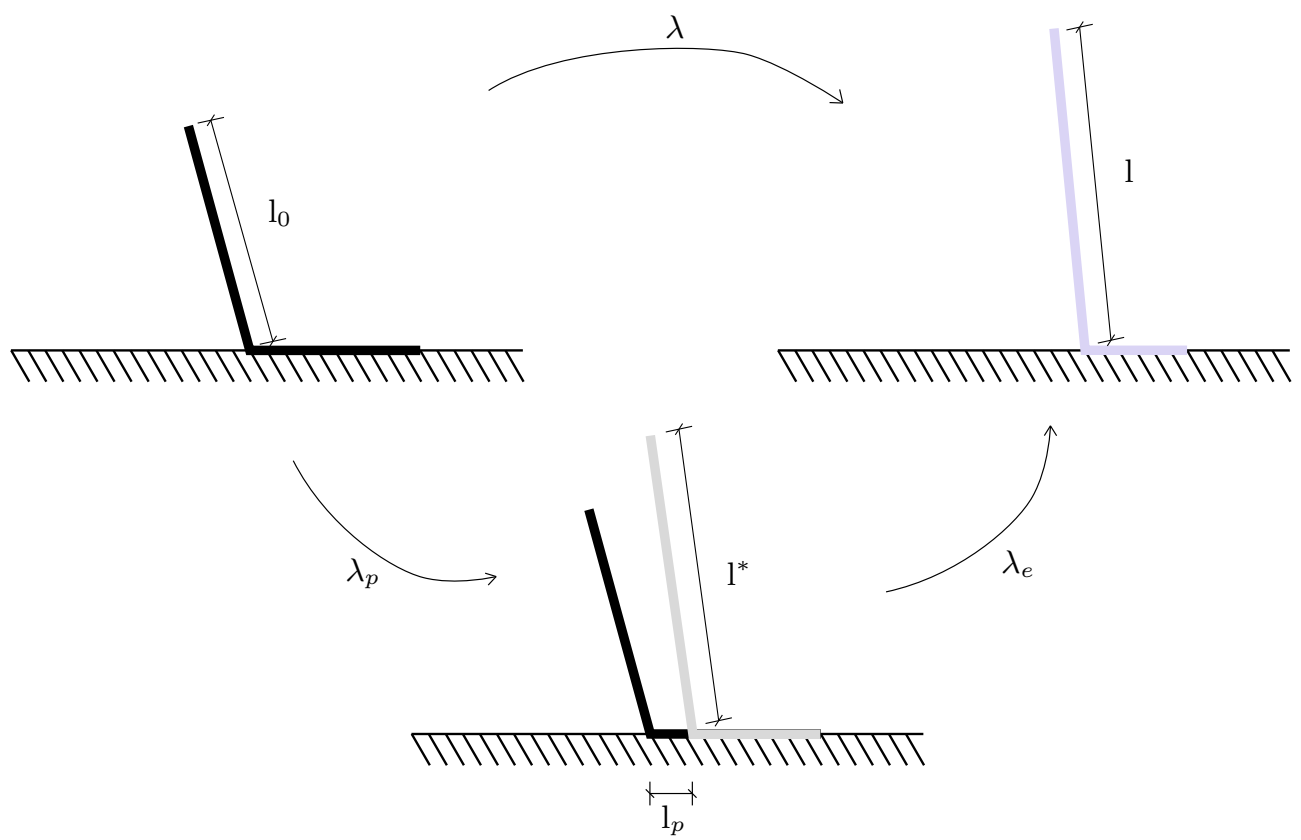

Fig. 3 Decomposition of the total stretch

where $l$ and $l_{0}$ are the current length and the initial length of the member, respectively. $l^{*}$ is the length in the stress-free intermediate configuration, and $l^{p}$ is the total peeling of the member. This is in fact the commonly used multiplicative decomposition in finite strain plasticity treatments. In the context of inelastic material modelling at finite strains, the deformation gradient $\mathbf{F}$, mapping the reference material vector to the current one is decomposed multiplicatively as $\mathbf{F}=\mathbf{F}_{e} \mathbf{F}_{p}$ where $\mathbf{F}_{p}$ is the plastic part of the deformation gradient and maps the material vector to the intermediate stress-free configuration representing the permanent changes at the microstructure, e.g. plastic slip. $\mathbf{F}_{e}$ is the part which deforms the material elastically and is the source of the stress developing at the material point under consideration. The evolution of the plastic part of the deformation is governed by a yield function (a higher-dimensional surface) and associated loading/unloading conditions which require dedicated update algorithms. A commonly used algorithm is known as the return mapping 
algorithm, where the material point under consideration is assumed to behave elastically (elastic predictor) under the action of incremental displacement/loading. Then, this assumption is checked by using the current status of the yield function, and if it is violated, the material state is projected onto the yield surface (plastic corrector). A detailed treatment of plasticity theories and the associated update algorithms can be found in [14] and [15].

Sticking to logarithmic strain definition, one can introduce

$$
\begin{aligned}
\ln \lambda & =\ln \lambda_{e}+\ln \lambda_{p}, \\
\epsilon & =\epsilon_{e}+\epsilon_{p},
\end{aligned}
$$

where $\epsilon_{p}$ is the permanent 'strain' developing in the member due to peeling. Obviously, this does not correspond to a physical strain since the microstructure of the fibre is not affected due to detachment from the substrate. Among various alternatives, by choosing the logarithmic strain as the deformation measure, the multiplicative description of the total stretch is converted into an additive form which is easier to treat.

Introducing a quadratic strain energy density in terms of elastic logarithmic strain, $\psi=1 / 2 E \epsilon_{e}^{2}$, and ignoring cross-sectional deformations, the strain energy of an element can be written as $\psi^{e}=1 / 2 E \epsilon_{e}^{2} A l$, where $A$ and $l$ are the cross-sectional area and the current length of the member, respectively. It has to be noted that the work conjugate stress measure to an increment of logarithmic strain is the Kirchhoff stress $\tau$. Therefore, the normal stress developing in the member is obtained as $\tau=E \epsilon_{e}$ where $E$ is the slope of $\tau$ versus logarithmic stress, a Young modulus-like quantity. In case of negligible cross-sectional deformations, Cauchy stress and Kirchhoff stress are related through $\tau=\lambda \sigma$ for an ordinary element and $\tau=\lambda_{e} \sigma$ for an element with a peeling end, since the peeling phase is volume preserving. Therefore, one can determine the Cauchy stress and in turn the internal force provided that $\epsilon_{e}$ is available.

Since the external loading is displacement driven, at element level, as proposed by Kendall [4], the evolution of peeling is governed by the balance between the change in strain energy due to a change in $l_{p}$ ( designated as $d l_{p}$ ) and the surface energy necessary to create a free surface of $2 b d l_{p}$ where $b$ is the width of the strip. Using $\psi^{e}$, the peeling condition can be expressed as

$$
\phi=-\frac{\partial \psi^{e}}{\partial l^{p}}-2 \gamma b \leq 0
$$

and put into the following explicit form:

$$
\phi=\tau A\left(\lambda_{e}-c\right)-\frac{1}{2} \tau \epsilon_{e} A c-2 \gamma b \leq 0 \quad \text { with } c=\mathbf{n}_{p} \cdot \mathbf{n}^{e} .
$$

A closer look at $c$ reveals that the inner product of the two unit vectors captures the angular orientation of the member with respect to the peeling direction. In Eq. (15), the first term is the work done by the already existing internal force $\tau A$ over $d l_{p}\left(\lambda_{e}-c\right)$, the second term is the strain energy stored in the projected peeled off length $c d l_{p}$, and the last term is the energy necessary to create a free surface of $2 b d l_{p}$. Although each term conforms to the energy balance arguments given by Kendall [4], in this elaboration, the orientation of the strip is captured in a natural way. Therefore, this form can be interpreted as the geometrically nonlinear version of the energy balance given by Kendall. It must be noted that the starting and end nodes for elements with a peeling end have to be chosen such that $\mathbf{n}_{e}=\frac{\mathbf{x}_{e}-\mathbf{x}_{s}}{\left\|\mathbf{x}_{e}-\mathbf{x}_{s}\right\|}$ should point to the peeling end.

Function $\phi$ is equivalent to yield functions of plasticity theories and the corresponding loading-unloading conditions can be introduced as

$$
\phi \leq 0, \quad i_{p} \geq 0, \quad \phi i_{p}=0,
$$

controlling the peeling evolution. Although there exists a strong analogy between plasticity and peeling, it must be noted that $l_{p}$ is not an internal variable.

A commonly used method to address inequality constraints is the active set algorithm which requires prediction and verification of a set of elements which are violating the constraint (in this context peeling criterion) in the increment under consideration. Based on this assumed active set, the iteration is completed. The configuration is updated with the iterative corrections and at this stage the assumed active set has to be verified. The elements of the set which do not 'peel' any more have to be dropped out, and elements which are 'activated' with the updated configuration have to be inserted in the active set. This method is used in rate-independent crystal plasticity [16] and in fact among commonly used numerical optimization techniques, [17]. However, prediction, verification and update of the active set is not a trivial task and might 
Table 1 Overall solution algorithm

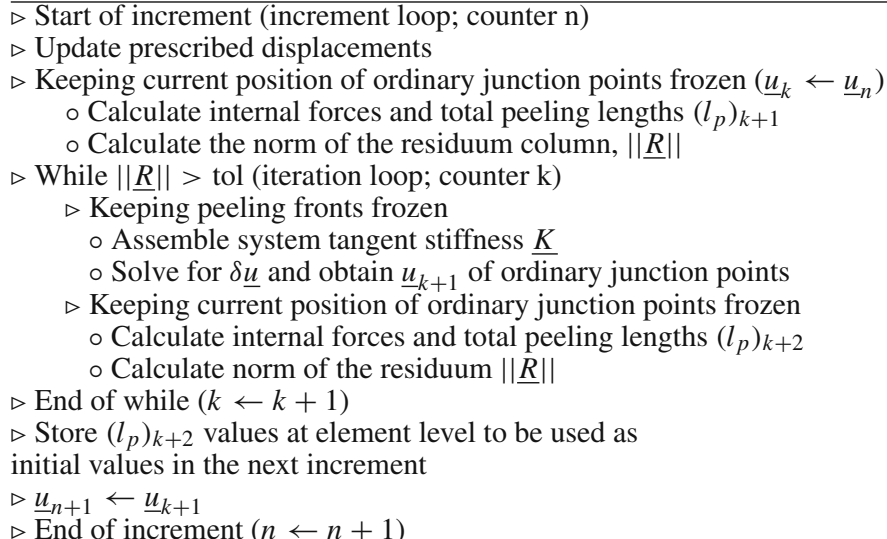

require problem-dependent adaptations. Therefore, in this work, a different route is taken which determines the system unknowns (displacements of ordinary junction points and $l_{p}^{e}$ 's) in a staggered way within an increment. Furthermore, the approach uses the structure of the return mapping algorithm as detailed in the next section.

\subsection{Solution procedure}

In an incremental-iterative setting, the prescribed displacements are updated at the beginning of the increment. Next, keeping the current position of ordinary junction points frozen, internal forces and updated total peeling lengths are calculated following the procedure explained in the next subsection. Using the updated total peeling lengths and Eq. (7), the current position of the peeling fronts and internal forces are determined. If the norm of the residuum column is larger than the pre-set threshold value (user-defined convergence criterion), element tangent stiffness matrices are evaluated with frozen peeling fronts and the system tangent stiffness is assembled. By solving the resulting system of equations (5), updated nodal displacements of ordinary points are obtained. With the updated nodal displacements, internal forces and total peeling lengths are calculated. This iterative procedure is repeated until the convergence criterion is met, and overall algorithm is summarized in Table 1. It must be noted that the solution algorithm is staggered within an increment.

In the next subsection, the return mapping algorithm used to calculate the peeling of each element is presented.

\subsection{Return mapping algorithm}

Referring to Table 1, after $\underline{u}_{k+1}$ is obtained, at element level, peeling length $\left(l_{p}\right)_{k+1}$, total stretch $\lambda_{k+1}$, total strain $\epsilon_{k+1}$, peeling stretch $\left(\lambda_{p}\right)_{k+1}$ and permanent strain $\left(\epsilon_{p}\right)_{k+1}$ are available. Assuming that there is no peeling evolution taking place, one gets the trial elastic stretch and strain using

$$
\lambda_{e, \text { trial }}=\frac{\lambda_{k+1}}{\left(\lambda_{p}\right)_{k+1}}, \quad \epsilon_{e, \text { trial }}=\epsilon_{k+1}-\left(\epsilon_{p}\right)_{k+1},
$$

and in turn the corresponding Kirchhoff stress using

$$
\tau_{\text {trial }}=E \epsilon_{e, \text { trial }}
$$

Plugging these results into the peeling criterion yields

$$
\phi_{\text {trial }}=A \tau_{\text {trial }}\left(\lambda_{e, \text { trial }}-c_{k+1}\right)-\frac{1}{2} A \tau_{\text {trial }} \epsilon_{e, \text { trial }} c_{k+1}-2 \gamma b,
$$


the trial peeling criterion which indicates that there is indeed no peeling evolution provided that $\phi_{\text {trial }} \leq 0$. Otherwise (if $\phi_{\text {trial }}>0$ ), further peeling takes place and the consistency condition $\phi^{k}=0$ is used to determine the current peeling length $\left(l_{p}\right)_{k+1}$. The consistency condition reads

$$
\begin{aligned}
\phi_{k+1}= & E A\left(\epsilon_{k+1}-\left(\epsilon_{p}\right)_{k+2}\right)\left(\frac{\lambda_{k+1}}{\left(\lambda_{p}\right)_{k+2}}-c_{k+2}\right) \\
& -\frac{1}{2} E A\left(\epsilon_{k+1}-\left(\epsilon_{p}\right)_{k+2}\right)^{2} c_{k+2}-2 \gamma b,
\end{aligned}
$$

which can be expressed solely in terms of $\left(l_{p}\right)_{k+2}$ and solved for it using a local Newton-Raphson algorithm. Dropping the subscripts $k+1$ and $k+2$ for the sake of clarity, the linearized form of Eq. (20) can be expressed and solved as

$$
\begin{aligned}
& \phi_{m}+\left.\frac{\partial \phi}{\partial l_{p}}\right|_{m} \delta l_{p}=0, \\
& \phi_{m}+g_{m} \delta l_{p}=0, \quad \delta l_{p}=-\phi_{m} / g_{m}, \quad\left(l_{p}\right)_{m+1}=\left(l_{p}\right)_{m}+\delta l_{p},
\end{aligned}
$$

where $g$ designates $\frac{\partial \phi}{\partial l_{p}}$ as obtained from Eq. (20), $\delta l_{p}$ is the iterative corrective term, and $m$ is the iteration counter of the local Newton-Raphson algorithm. The converged value of $l_{p}$ corresponds to $\left(l_{p}\right)_{k+2}$.

Referring back to the equilibrium equations of the system (5) since the solution is obtained by the NewtonRaphson algorithm, consistent linearization is essential. The details of linearization for ordinary members are given in reference [11].

For elements with peeling end, the material tangent $T$ simply reduces to

$$
T=\frac{d \tau_{n+1}}{d \epsilon_{n+1}}=E
$$

provided that $\phi_{n+1}^{\text {trial }}<0$. Otherwise, the corresponding total differential reads

$$
\frac{d \tau_{n+1}}{d \epsilon_{n+1}}=\frac{d \tau_{n+1}^{\mathrm{trail}}}{d \epsilon_{n+1}}-E \frac{d \Delta \epsilon^{p}}{d \epsilon_{n+1}}=E\left(1-\frac{d \Delta \epsilon^{p}}{d \epsilon_{n+1}}\right),
$$

where $\Delta \epsilon_{p}=\ln \frac{l_{n+1}^{*}}{l_{n}^{*}}$. In order to obtain $d \Delta \epsilon^{p} / d \epsilon_{n+1}$, the persistency condition (steady satisfaction of the peeling function) $\dot{\phi}=0$ is used, which results in

$$
\dot{\phi}=\left[\left.\frac{\partial \phi}{\partial \epsilon}\right|_{\epsilon} p+\left.\frac{\partial \phi}{\partial l_{p}}\right|_{\epsilon} \frac{\partial l_{p}}{\partial \epsilon}\right] \dot{\epsilon},
$$

implying

$$
\frac{\partial\left(l_{p}\right)_{n+1}}{\partial \epsilon_{n+1}}=-\left.\frac{1}{l_{n+1}^{*} g} \frac{\partial \phi_{n+1}}{\partial \epsilon_{n+1}}\right|_{\left(l_{p}\right)_{n+1}} .
$$

$\frac{\partial \phi_{n+1}}{\partial \epsilon_{n+1}}$ at fixed $\left(l_{p}\right)_{n+1}$ can be obtained through $\frac{\partial \phi_{n+1}}{\partial \epsilon_{n+1}}=\left.\frac{\partial \phi_{n+1}}{\partial\left(\lambda_{e}\right)_{n+1}}\right|_{\epsilon_{n+1}} \frac{\partial\left(\lambda_{e}\right)_{n+1}}{\partial \epsilon_{n+1}}$ in a straightforward manner.

\section{Numerical examples}

The proposed framework is implemented in MATLAB and numerical examples are presented in the following subsections. As mentioned in Sect. 2, the formulation and algorithm does not allow members to be in compression. In the presented examples, such cases are not confronted with. As far as convergence of the computations is concerned, the $L_{2}$ norm (Eucledian norm) of the residuum column is compared with the convergence tolerance of $10^{-8}$. The loading is displacement controlled, and the presented examples could be completed without any difficulty. 


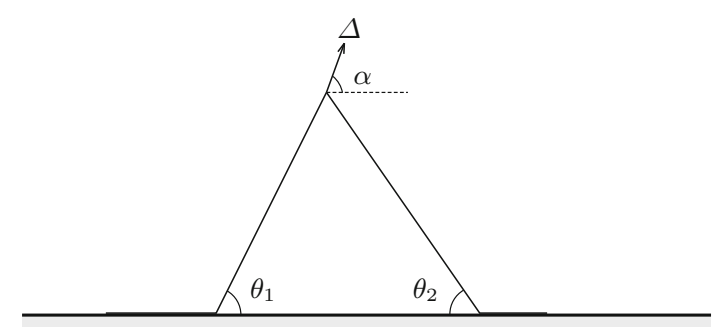

Fig. 4 Geometric layout for double-peeling problem

Table 2 Parameter set for double-peeling problem (top) and for staple pin anchorage problem (bottom)

\begin{tabular}{llll}
\hline & Case I & Case II & Case III \\
\hline $\mathrm{E}(\mathrm{Mpa})$ & 3 & 3 & 3 \\
$\mathrm{~J}\left(\mathrm{~N} / \mathrm{mm}^{2}\right)$ & 0.01 & 0.01 & 0.01 \\
$\theta_{1}\left({ }^{\circ}\right)$ & 90 & 0 & 60 \\
$\theta_{2}\left({ }^{\circ}\right)$ & 90 & 0 & 60 \\
$\alpha\left(^{\circ}\right)$ & 90 & 90 & 45 \\
$\mathrm{l}_{1}(\mathrm{~mm})$ & 10 & 10 & 10 \\
$\mathrm{l}_{2}(\mathrm{~mm})$ & 10 & 10 & 10 \\
$\Delta(\mathrm{mm})$ & 50 & 50 & $50 \sqrt{2}$ \\
\hline $\mathrm{E}_{l}(\mathrm{Mpa})$ & 3 & & \\
$\mathrm{E}_{b}(\mathrm{Mpa})$ & 3 & & \\
$\mathrm{~J}\left(\mathrm{~N} / \mathrm{mm}{ }^{2}\right)$ & 0.01 & & \\
$\mathrm{~s}(\mathrm{~mm})$ & 15 & & \\
$1(\mathrm{~mm})$ & 1 & & \\
$\alpha\left({ }^{\circ}\right)$ & 90 & & \\
$\Delta(\mathrm{mm})$ & 50 & & \\
\hline
\end{tabular}

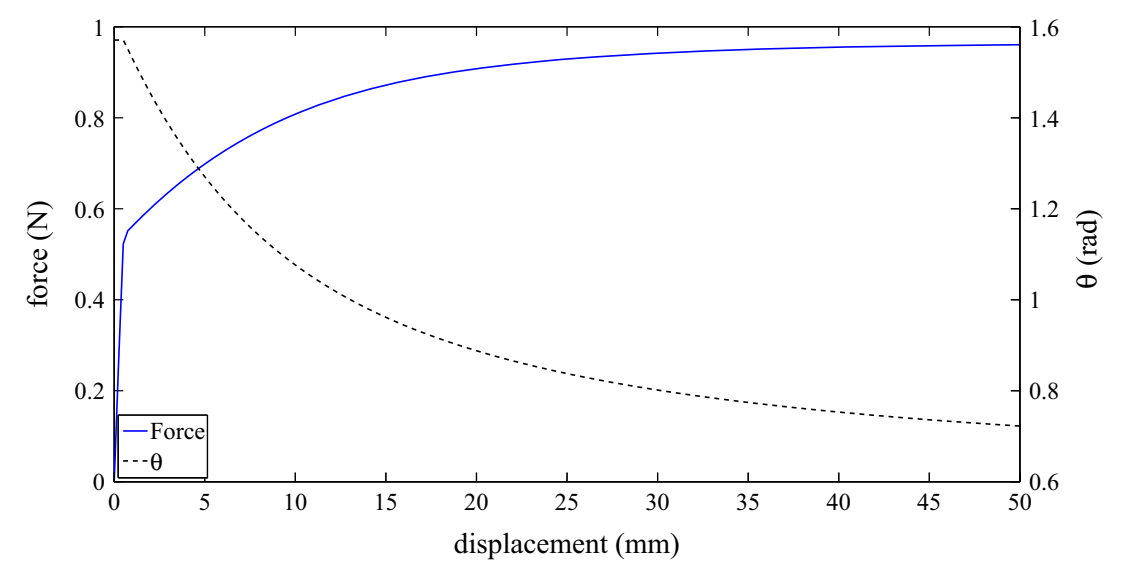

Fig. 5 Evolution of peeling force and angle for case I. Due to symmetry, magnitudes of $\theta_{1}$ and $\theta_{2}$ are equal and designated as $\theta$

\subsection{Double peeling}

In this example, entire detachment process of a 2-D structure consisting of two strips is considered as shown in Fig. 4. This problem was addressed by [10] and is used here as the basis of verification of the current formulation. The parameters of the system are taken from the aforementioned reference and given on the top part of Table 2. Three different cases, labelled as case I, case II and case III, are considered and explained below.

In the first case, a symmetrical initial configuration $\left(\theta_{1}=90^{\circ}, \theta_{2}=90^{\circ}\right.$ and $\left.\alpha=90^{\circ}\right)$ is analysed. As seen in Fig. 5, both the peeling force and the peeling angle are approaching to limiting values as the prescribed displacement increases. The geometry of the structure approaches, at a rather slow rate, to the optimum configuration as the peeling force approaches its maximum in a similar way. However, if both angles $\theta_{1}$ and 


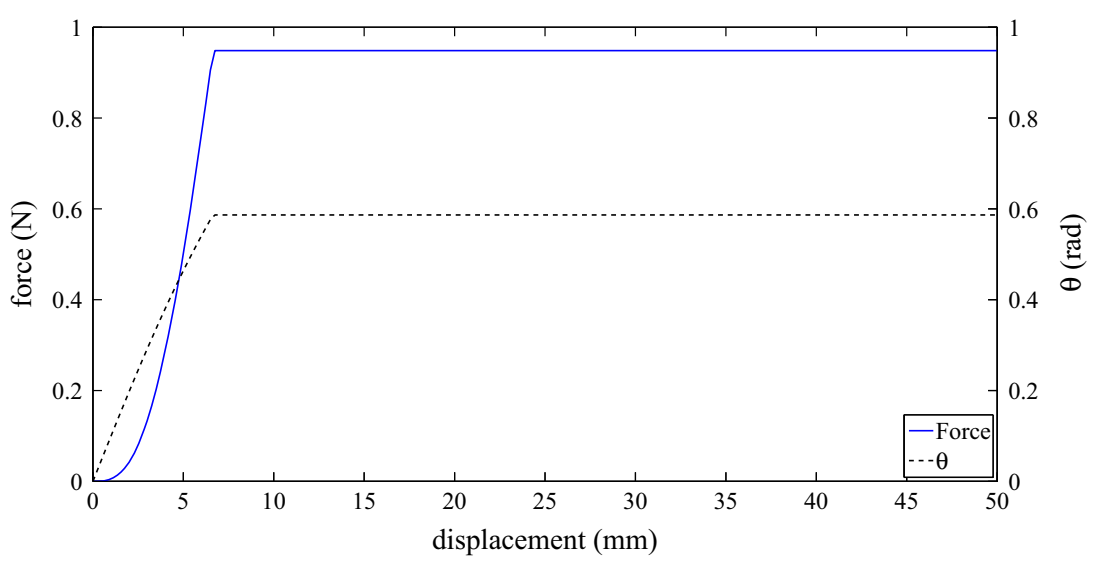

Fig. 6 Evolution of peeling force and angle for case II. Due to symmetry, magnitudes of $\theta_{1}$ and $\theta_{2}$ are equal and designated as $\theta$

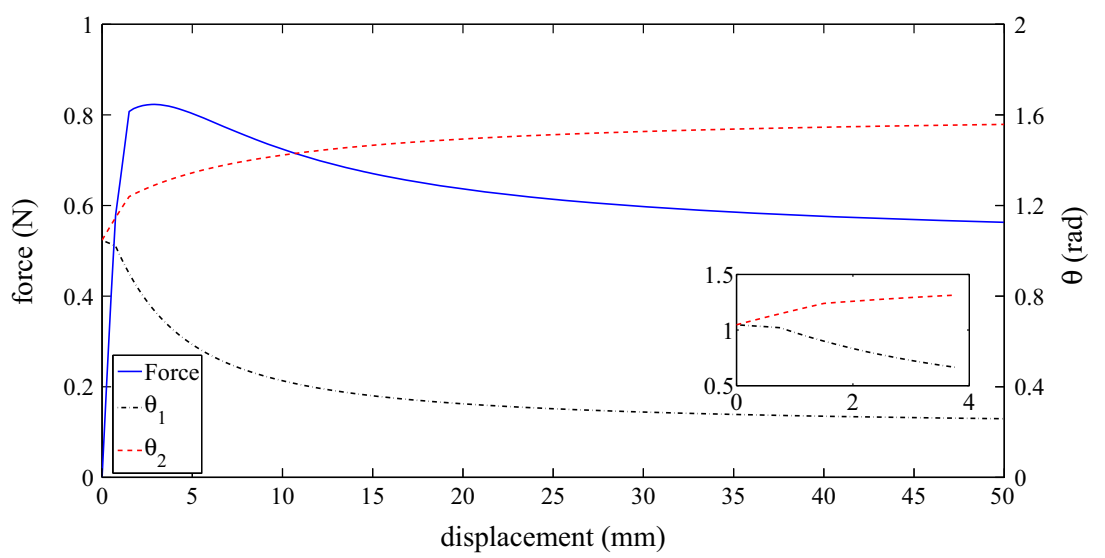

Fig. 7 Evolution of peeling force and angles for case III

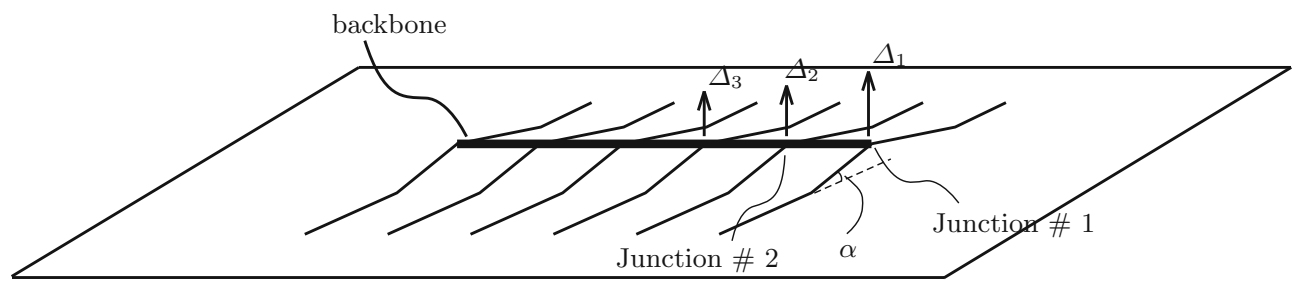

Fig. 8 Staple pin geometry

$\theta_{2}$ are set to zero in the initial configuration (case II), abrupt changes in peeling force and peeling angle are observed as seen in Fig. 6. As discussed by the authors of [10], these constant values indicate that the structure has reached its optimum configuration. In case III, the prescribed displacement is applied with an angle of $\alpha=45^{\circ}$ for which the peeling force is shown in Fig. 7. From the evolution of angles as shown in Fig. 7, it is clear that the geometry of the two strips evolves in dissimilar ways. The inset in Fig. 7 shows that the onset of peeling takes place at different displacement levels which results in a kink in the 'elastic' branch of the force-displacement graph also shown in Fig. 7.

Qualitatively, all of these results are in full agreement with the results presented in [10]. The peeling force and angle values are close but not identical to the values of reference [10] since stresses are based on logarithmic strains and orientation-sensitive peeling criterion is used in this work. 


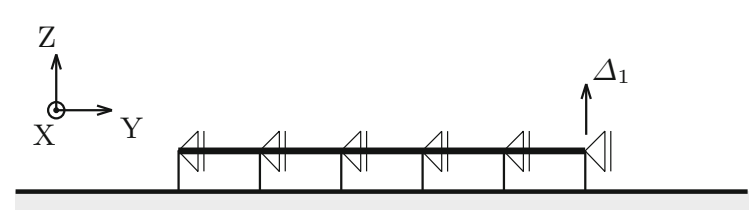

Side view

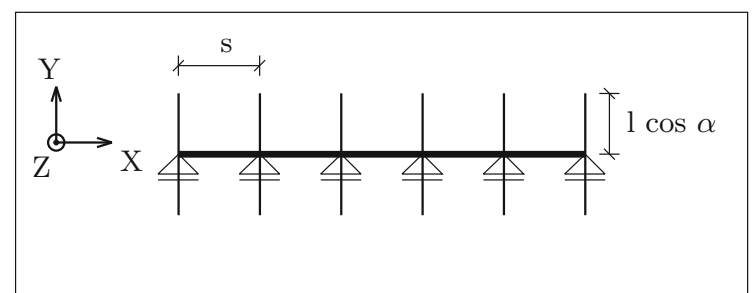

Top view

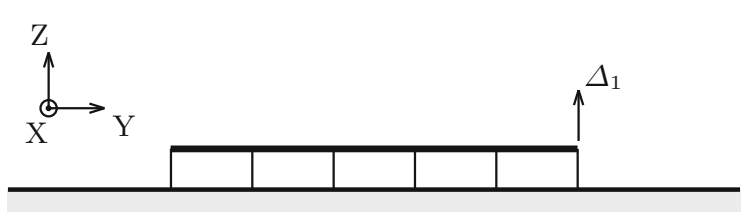

Side view

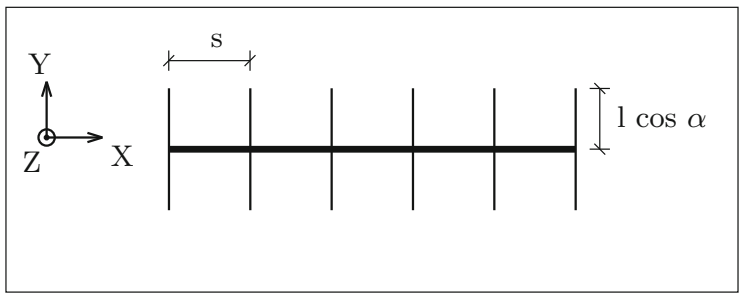

Top view

Fig. 9 Two different sets of boundary conditions imposed on the staple pin geometry; left is designated as BC I and the right as BC II
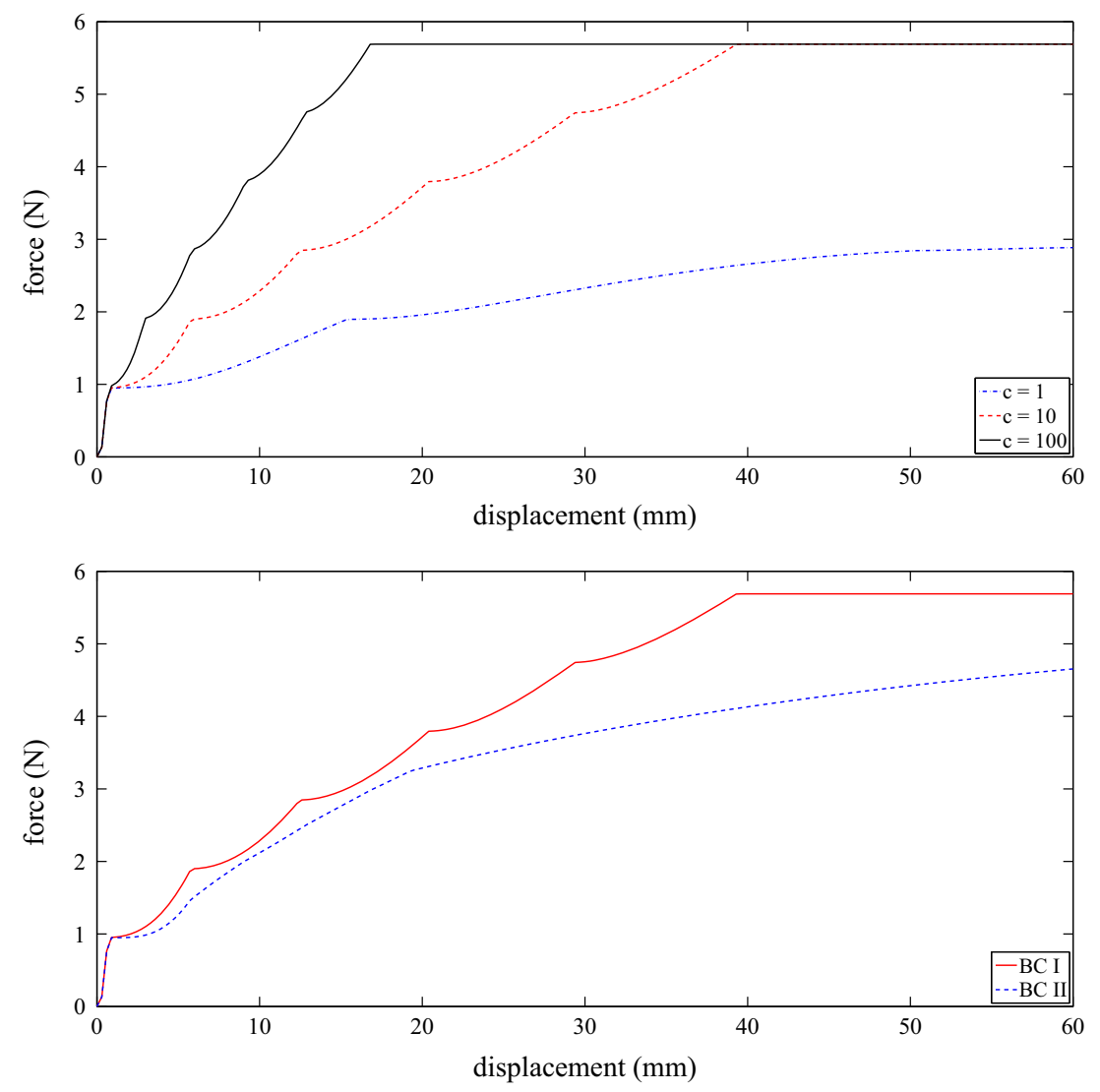

Fig. 10 Force-displacement response for different case—top : effect of backbone stiffness; bottom: effect of boundary conditions

\subsection{Multiple peeling in stable pin geometry}

Spider anchorages have essentially two different geometric forms with different functions. The layout considered in this example is called as 'staple pin' structure, and an idealized geometry is shown in Fig. 8. The initial angle of lateral strips is close to zero, and they typically branch off a much stiffer major component. To analyse 

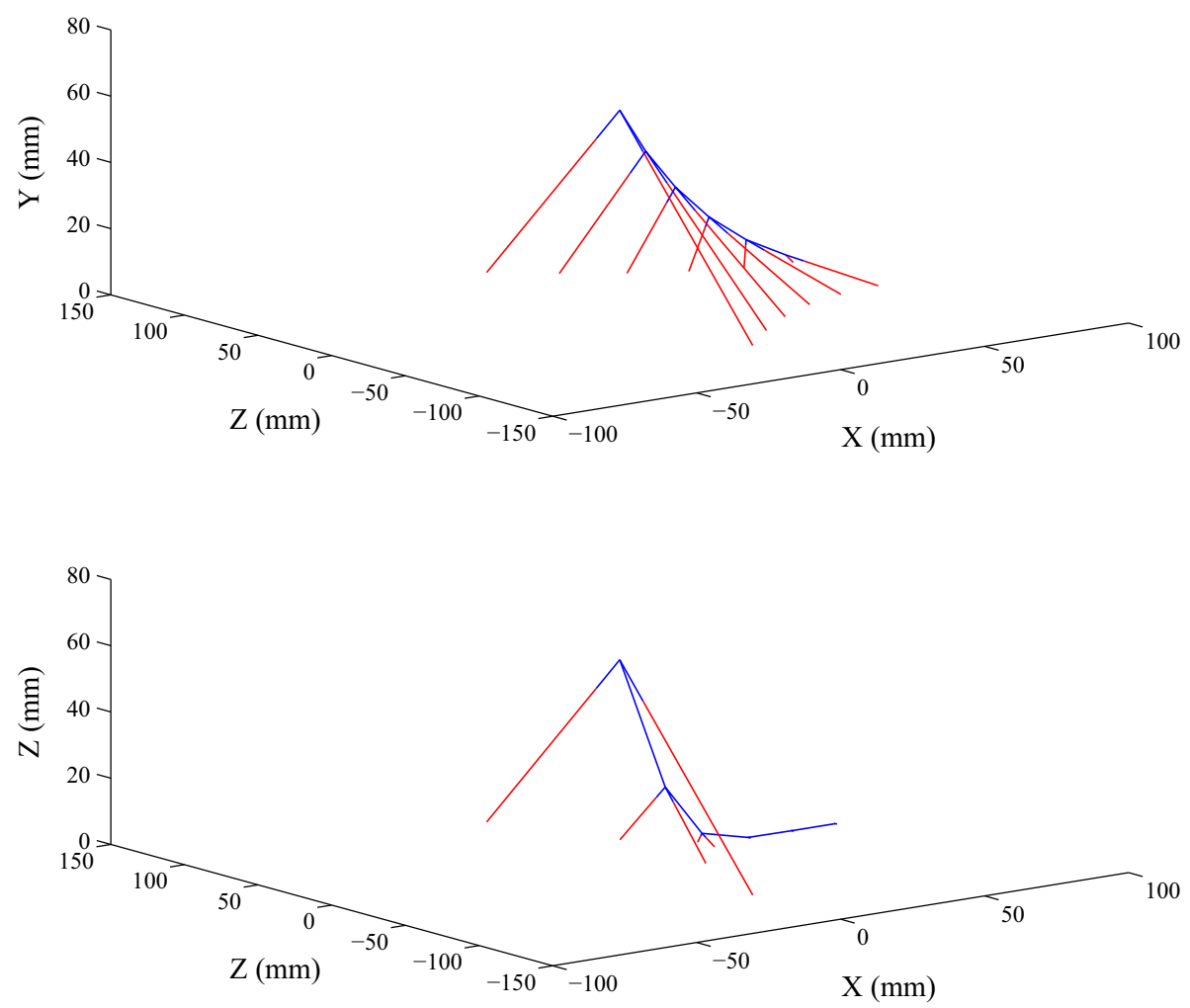

Fig. 11 Deformed configuration for two different contrast values; top $c=100$ and bottom $c=1$ Red portions represent the peeled lengths

the detachment process of such a structure, a geometric model composed of 6 pairs of lateral branches and a backbone element is constructed for which the parameters are summarized on the bottom part of Table 2 .

Obviously, the structure is composed of a number of V-shaped double-strips branching off the stiffer backbone. The solution presented in reference [10] uses a set of prescribed displacements imposed on junction points of $\mathrm{V}$ strips which are linearly dependent on the displacement imposed on the first junction point, please see Fig. 8. Consequently, their solution turns out to be the superposition of V-shaped symmetric double-peeling solutions and yields a step-like force-displacement response. It must be noted that these boundary conditions are not realistic.

By imposing the boundary conditions shown on the left-hand side of Fig. 9 ( $X$ and $Y$ displacements of the junction points of V strips are constrained, and these boundary conditions are designated as BC I), a very similar problem (to the one presented in reference [10]) is constructed in which the displacements of the second and the following junction points are not prescribed. Three different cases with three different backbone stiffnesses are considered. Defining $c=\frac{E_{\text {aackbone }}}{E_{\text {lateral }}}$ (the contrast value), force-displacement response for three different cases is presented in Fig. 10. For $c=10$ and $c=100$, the step-like behaviour (with relatively short plateaus) is clearly visible. Having a stiffer backbone results in a much faster evolution of the peeling, whereas in case of equal stiffness $(c=1)$, the peeling of just the front three pairs is activated and the displacement is accommodated largely by peeling of the two front members and elastic deformation of the backbone portion connecting junction points 1 and 2 as shown in Fig. 11.

A more realistic case with regard to boundary conditions is obtained when all of the restraints are removed, please see boundary conditions BC II in Fig. 9. In this case, the interaction between consecutive V strips is activated, and this results in force-displacement curve shown in Fig. 10. The peak force decreases significantly (approximately 17\% drop in peak force), and the step-like shape of the curve disappears, and the response gets softer. 

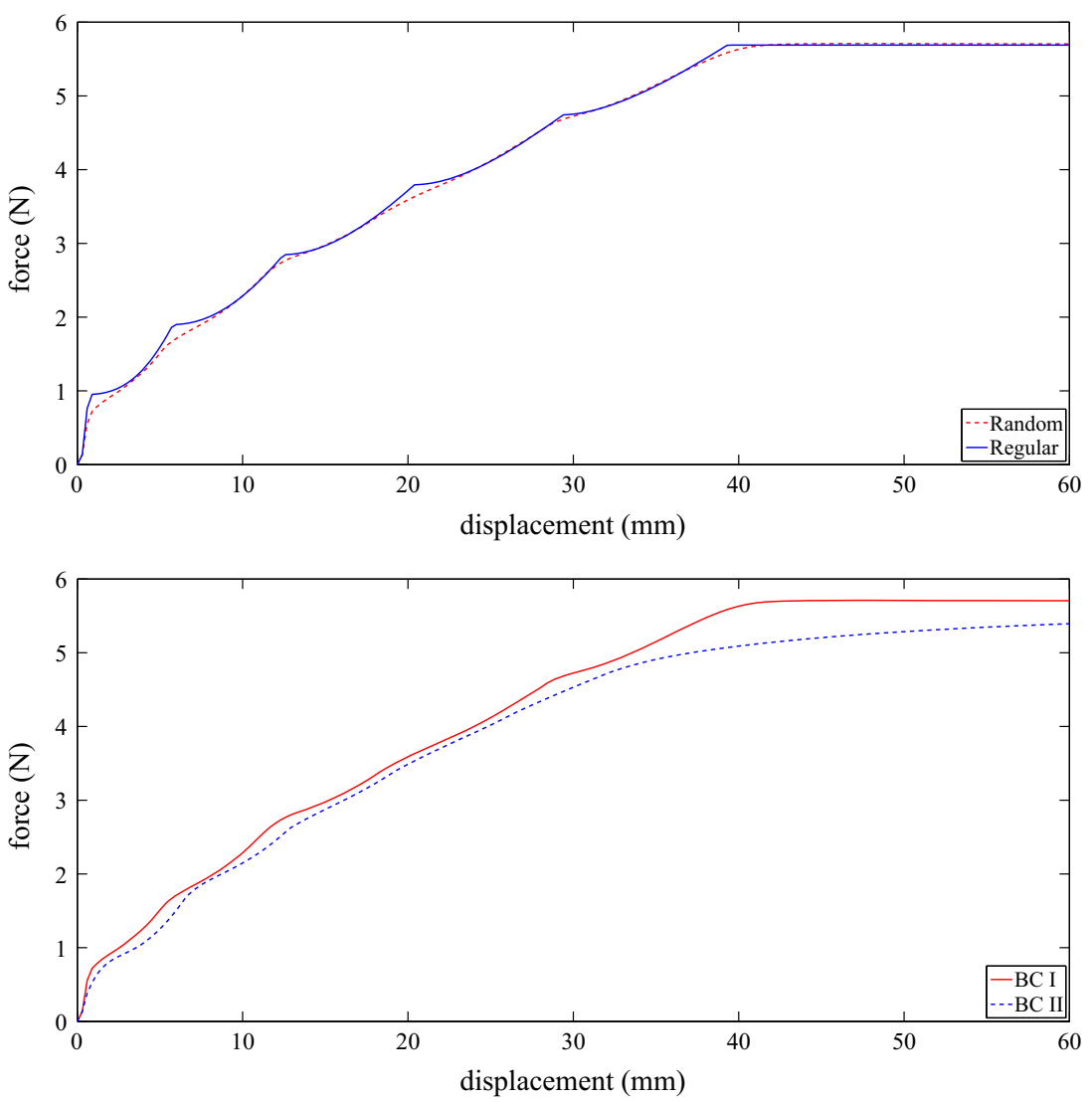

Fig. 12 Effect of random orientation of the lateral strips-top: regular and random structures under identical boundary conditions (BC I); bottom: random structure under different boundary conditions

Micro-graphs of spider anchorages reveal that in reality, the lateral strips are typically attached to the substrate at different angles. In other words, the peeling directions are not parallel to each other. To mimic this feature, a model with random in-plane peeling directions is constructed. Therefore, this results in a model where the lateral strips are not necessarily parallel to each other, but initial lengths of the lateral strips are kept the same.

Force-displacement curve of the structure with randomly oriented lateral strips under BC I is compared with that of the regular structure under the same boundary conditions in Fig. 12. Obviously, the kinks are smoothed out, whereas the same peak load is reached in both systems. To assess the influence of boundary conditions on model with randomly oriented strips, this structure is analysed under BC I and BC II, respectively. The corresponding force-displacement curves are shown in Fig. 12. Under BC II, the peak force gets smaller (a comparable trend with regular structure, please see Fig. 10) and the step-like nature of the curve is still visible at least in the initial phase of the peeling process.

\section{Conclusion and outlook}

In this paper, multiple peeling of 3D arbitrarily oriented elastic strips is addressed in a computational setting. Using a geometrically nonlinear mechanics framework, deformation of strips with peeling ends are decomposed multiplicatively akin to decomposition used in finite strain inelasticity formulations. Furthermore, by treating energy balance as the peeling criterion as suggested by Kendall [4], a return mapping algorithm is introduced and the peeling front of each strip is effectively determined. Adopting a staggered solution algorithm at the global level, current configuration of peeling fronts and other nodes/junction points is updated consecutively.

The performance of this approach is demonstrated by a set of example problems. Results of these numerical tests suggest that the use of superposition as suggested by [10] is limited particularly under realistic boundary conditions. 
The members which might step into the compression region are not considered in this work and can be considered as a potential extension in the future within a dynamic relaxation-type solution framework. Under non-monotonic loading conditions, the performance of the staggered solution might deteriorate and calls for further testing under such loading histories. This has to be done carefully since the members are of tension-only nature and some non-monotonic loading patterns might push the structure into unstable configurations. An interesting extension would be the inclusion of surface pattern in this framework which seems to be tractable due to explicit appearance of peeling direction in the formulation. Furthermore, the multiplicative decomposition framework can be exploited effectively to treat peeling of inelastic strips. For relatively thicker strips, potential use of the presented approach in the context of beam-type structural elements can be pursued in order to take bending stiffness into account. Finally, the presented framework could potentially be used within a multiscale modelling framework addressing the failure of adhesive interfaces.

\section{References}

1. Arzt, E., Gorb, S., Spolenak, R.: From micro to nano contacts in biological attachment devices. Proc. Natl. Acad. Sci. U. S. A. (PNAS) 100(19), 10603-10606 (2003)

2. Pugno, N.M., Cranford, S.W., Buehler, M.J.: Synergetic material and structure optimization yields robust spider web anchorages. Small 9(16), 2747-2756 (2013)

3. Sahni, V., Harris, J., Blackledge, T.A., Dhinojwala, A.: Cobweb-weaving spiders produce different attachment discs for locomotion and prey capture. Nat. Commun. 3, 1106 (2012)

4. Kendall, K.: Thin-film peeling — the elastic term. J. Phys. D Appl. Phys. 8, 1449-1452 (1975)

5. Chen, B., Wu, P., Gao, H.: Hierarchical modelling of attachment and detachment mechanisms of Gecko toe adhesion. Proc. R. Soc. A 464, 1639-1652 (2008)

6. Chen, B., Wu, P., Gao, H.: Pre-tension generates strongly reversible adhesion of a spatula pad on substrate. J. R. Soc. Interface 6, 529-537 (2009)

7. Pugno, N.M.: The theory of multiple peeling. Int. J. Fract. 171, 185-193 (2011)

8. Afferante, L., Carbone, G., Demelio, G., Pugno, N.: Adhesion of elastic thin films: double peeling of tapes versus axisymmetric peeling of membranes. Tribol. Lett. 52, 439-447 (2013)

9. Bosia, F., Colella, S., Mattoli, V., Mazzolai, B., Pugno, N.M.: Hierarchical multiple peeling simulations. RSC Adv. 4, 25447-25452 (2014)

10. Brely, L., Bosia, F., Pugno, N.M.: Numerical implementation of multiple peeling theory and its application to spider web anchorages. Interface Focus 5, 20140051 (2015)

11. Bonet, J., Wood, R.D.: Nonlinear Continuum Mechanics for Finite Element Analysis, 2nd edn. Cambridge University Press, Cambridge (2015)

12. Krenk, S.: Nonlinear Modeling and Analysis of Solids and Structures. Cambridge University Press, Cambridge (2009)

13. Wriggers, P.: Nonlinear Finite Element Methods. Springer-Verlag, Berlin Heidelberg (2008)

14. Simo, J.C., Hughes, T.J.R.: Computational Inelasticity. Springer-Verlag, Berlin Heidelberg (1998)

15. de Souza Neto, E.A., Peric, D., Owen, D.R.J.: Computational Methods for Plasticity. Wiley, Hoboken (2008)

16. Anand, L., Kothari, M.: A computational procedure for rate-independent crystal plasticity. J. Mech. Phys. Solids 44(4), $525-558(1996)$

17. Nocedal, J., Wright, S.J.: Numerical Optimizaiton, 2nd edn. Springer-Verlag, Berlin, New York (2006) 